\title{
Gradient- and spin-echo (GRASE) MR imaging: a long-existing technology that may find wide applications in modern era
}

\author{
Mei-Lan Chu ${ }^{1,2}$, Cheng-Ping Chien ${ }^{1,3}$, Wen-Chau Wu ${ }^{4}$, Hsiao-Wen Chung ${ }^{1}$ \\ ${ }^{1}$ Graduate Institute of Biomedical Electronics and Bioinformatics, National Taiwan University, Taipei, Taiwan; ${ }^{2}$ Department of Biomedical Sciences \\ and Engineering, National Central University, Taoyuan, Taiwan; ${ }^{3}$ Department of Radiology, Taipei Beitou Health Management Hospital, Taipei, \\ Taiwan; ${ }^{4}$ Institute of Medical Device and Imaging, National Taiwan University, Taipei, Taiwan
}

Correspondence to: Hsiao-Wen Chung, PhD. Professor, Graduate Institute of Biomedical Electronics and Bioinformatics, National Taiwan University, Taipei, Taiwan. Email: chunghw@ntu.edu.tw.

Submitted Sep 01, 2019. Accepted for publication Sep 10, 2019.

doi: 10.21037/qims.2019.09.13

View this article at: http://dx.doi.org/10.21037/qims.2019.09.13

\section{Introduction}

There are plenty of technical innovations that were regarded in early days as certainly impractical during initial usage, yet later proved to become the predominant method of choice in certain applications after key improvements of the associated equipment or devices. Well known examples in the magnetic resonance (MR) imaging literature include clinical diffusion-weighted imaging (1) that relies largely on the commercialization of single-shot echo-planar imaging (EPI) (2) which, in turn, requires high-performance gradient coils to reduce artifacts related to off-resonance to diagnostically acceptable level (3). Another instance is balanced steady-state free precession imaging, whose banding artifacts precluded its inclusion in routine clinical examination protocols before the advancements of gradient coils and shimming technology (4), but nowadays has become an essential tool in cardiovascular MR imaging (5). The gradient- and spin-echo (GRASE) sequence $(6,7)$ in our opinion, although not as obvious as the two aforementioned examples, may well be another imaging technique with strong potential but was long overlooked.

\section{Principles and properties of the GRASE sequence}

The pulse sequence diagram of the GRASE sequence is schematically shown in Figure $1(6,7)$. It consists of multiple refocusing radiofrequency (RF) pulses after the excitation RF pulse just like the fast spin-echo (FSE) technique (8), but with series of gradient-echo readouts in a way similar to EPI inserted in the echo spacing interval between successive refocusing pulses. Each gradient-echo is preceded by appropriate phase encoding gradients (not shown) to be filled in the k-space at corresponding locations to form an image. Therefore the GRASE sequence can be viewed as a hybrid of FSE and EPI.

Originally developed nearly thirty years ago when highperformance gradient coils were still uncommon in clinical whole-body MR imaging systems and hence EPI was a luxury, GRASE was proposed as a practically feasible fast sequence offering single-shot capability with T2-weighted contrast. Compared with EPI, the formation of multiple spin echoes in GRASE allows more k-space lines to be sampled with each excitation RF pulse. Single-shot GRASE imaging within one or two $\mathrm{T} 2$ relaxation times was thus possible for one two-dimensional (2D) slice, even with conventional gradient coils at a maximal strength of $10 \mathrm{mT} / \mathrm{m}$ commonly used in the 90s (9).

The hybrid characteristics of GRASE certainly lead to the advantages of speeded acquisition from EPI as well as reduced sensitivity to off-resonance effects from FSE, yet also accompanied by inevitable shortcomings from both techniques. Taking single-shot 2D GRASE as an example, phase errors induced by field inhomogeneity accumulate during the multiple gradient-echo readout as in EPI, and refocused at the center of each spin-echo. This, along with the transverse relaxation effects, results in periodic modulation of the echo signals in the $\mathrm{k}$-space if linear 


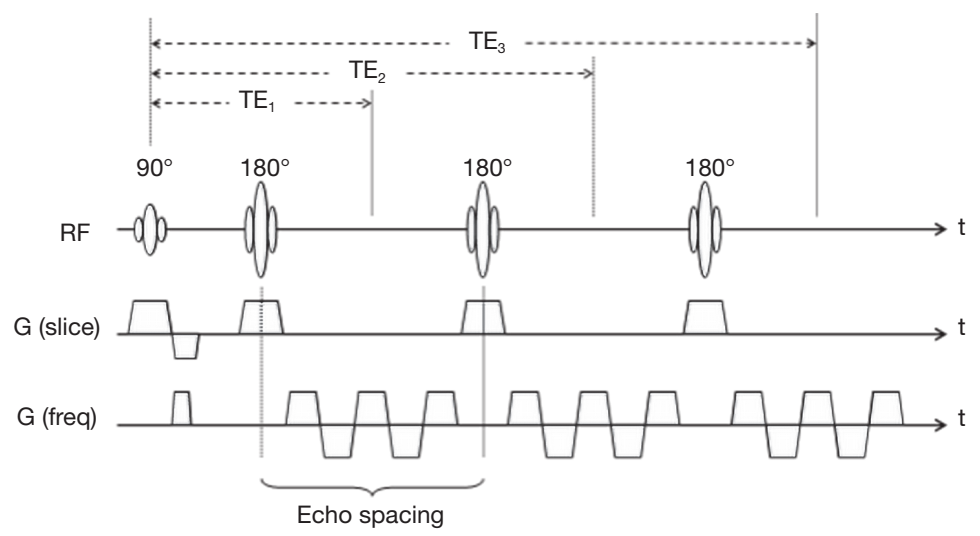

Figure 1 Schematic drawing of the pulse sequence diagram for the GRASE sequence, including the RF pulse, the slice selection gradient, and the frequency-encoding gradient channels. A number of refocusing pulses (three in this example) are applied after the excitation pulse to generate spin echoes at different TEs, whereas within each echo spacing a series of gradient-echoes (five here) are formed to sample the spin echo. Note that phase accumulation from off-resonance effects occurs within each spin echo, whereas signal decaying from T2 relaxation causes amplitude modulations across the spin echoes. GRASE, gradient- and spin-echo; RF, refocusing radiofrequency; TE, echo time.

phase encoding is used. Point-spread-function-related ghosting artifacts thus appear in the reconstructed image along the phase-encoding direction (10). Interestingly, note that T2-related amplitude oscillations and off-resonance phase modulations are not synchronous with each other. Consequently, although one could, for example, reduce phase-related ghosts via appropriate shuffling of the echo signals by varying the order of phase encoding gradients, residual amplitude oscillations are unavoidable (11). Variations of the phase encoding schemes to minimize artifact, therefore, were once an active topic of investigations for GRASE-related imaging techniques (12).

The previous explanations about ghosting artifacts in GRASE imaging are for single-shot 2D imaging only. With multi-shot GRASE, the above issues could be effectively alleviated with proper arrangement of the phase encoding order. This is however achieved at the expense of scan time penalty, where a long repetition time (TR) is likely needed to allow for full $\mathrm{T} 1$ recovery. Unfortunately in such a case GRASE often compares unfavorably with multi-shot FSE which has no phase error problems, as will be seen in a later section.

\section{Clinical evaluations of GRASE in early days}

The most frequently employed comparison target for GRASE was FSE, which had essentially replaced conventional spin-echo as the new standard for T2-weighted imaging as soon as it appeared in the market. In a majority of investigations reported for brain imaging at 1.5 Tesla and below, GRASE has been regarded as being inferior to FSE on aspects well explained by the aforementioned characteristics (13-15). Diminished contrast and lower signal-to-noise ratio (SNR) was reported for GRASE as compared with FSE (13), leading to inferior lesion conspicuity of GRASE (14). Even with reordered phase encoding to minimize ghosting artifacts, residual pointspread-functional blurring still resulted in substantially reduced sensitivity in GRASE for detecting small lesions (15). GRASE did occasionally show some advantages compared with FSE, for instance at depicting lesions with paramagnetic susceptibility characteristics like hemorrhage (16). Such a property is clearly understandable due to the gradient-echo nature of GRASE, which is again accompanied by gradientecho pitfalls such as increased chemical shift displacement artifacts along the phase encoding direction (17), and the advantage may not be applicable at lower field strengths due to reduced susceptibility effects (18). To make things worse, setting the clinical protocols to include a morphological FSE sequence plus a purely gradient-echo-based sequence would satisfy the requirements of reliable lesion depiction and hemorrhagic sensitivity (19), making GRASE out of the routine needs.

For body imaging, similar conclusions of the superiority of FSE to GRASE have been documented. In general, the overall image quality was regarded as significantly lower in GRASE than in FSE (20). Contrast-to-noise ratio for GRASE was also reported to compare inferiorly to FSE 
despite similar ability in tumor delineation (21). Since diagnostic accuracy certainly outweighs all other issues in clinical routine examinations, the role that can be played by GRASE became diminished with the attractive stability of image quality that can be brought about by the FSE technique.

On the other hand, the battle between GRASE and EPI underwent a completely different scenario. Whole-body high-performance gradient coils on clinical MR imaging systems started to appear in about 1994, making singleshot EPI a reality. Together with important advantages of diffusion-weighted readout exhibiting strong potential in the diagnosis of acute ischemic stroke $(22,23)$ and multislice capability free from cross-talk from the refocusing RF pulses (24), single-shot EPI soon found its unique role in clinical neural examinations, making comparative investigations between GRASE and EPI unnecessary.

\section{Unique advantages of GRASE}

Despite of the numerous negative reports mentioned in the previous section, GRASE does have its unique superiority in modern era. Specifically, high-field MR imaging systems (3.0 Tesla and above) begin to dominate technical advances in major manufacturers, and are more and more popular in medical centers around the world due to advantages brought about by improved SNR and spectral resolution. The two major competitors of GRASE, namely FSE and EPI as mentioned previously, have to face their own obstacles at high fields. The use of multiple refocusing pulses in FSE results in increased RF specific absorption rates (SAR), which worsens as the main field gets higher (25). The SAR limits the minimum echo spacing in FSE, which in turn lengthens the total acquisition time that could hurdle three-dimensional (3D) examinations within one breath-hold (26). For EPI on the other hand, geometric distortions worsen tremendously at high fields because the susceptibility effect scales more than linearly with field strength (27). The shortened $\mathrm{T} 2^{*}$ at high fields further places strong demands on fast switching gradient coils at high amplitudes. Suitability of GRASE imaging at high fields thus emerges $(28,29)$.

3D MR imaging at high fields is a good example where the GRASE sequence could exert its unique advantages. With 3D imaging, two loops of phase encoding are present. Smart reordering of the phase encoding steps allows a separation of phase error and T2 modulation in the k-space (10), hence permitting appropriate manipulation and filtering of the $\mathrm{k}$-space data differently along the two phase-encoding directions to minimize artifacts (30). T2related blurring can be further reduced using variable flip angle for the refocusing pulses (31) in a way to incorporate stimulated echo signals into the echo train (32). Along with the multiple gradient-echoes that effectively increase acquisition efficiency, 3D imaging at reduced scan time with stability approaching that of FSE becomes a possibility at high field strengths. In the following section, some current and foreseeable applications of GRASE imaging are described.

\section{Current and potential applications of GRASE imaging}

Like many other MR imaging sequences, GRASE can be used as a signal readout module with image contrast determined mainly in the preceding magnetization preparation stage, or as a standalone sequence whose diagnostic information relies on the evolution of magnetization during the relaxation process. In both cases, the unique characteristics of GRASE, namely reduced SAR compared with FSE and the better immunity to off-resonance than EPI, makes it ideally suited at high field strengths.

One area in which many investigators involved in technical developments of MR imaging prefer to utilize 3D GRASE as the signal readout module is arterial spin labeling (ASL) for perfusion imaging with RF-tagged intrinsic tracer. ASL is a typical example showing suitability for high-field imaging (e.g., 3.0 Tesla), due to the increased SNR as well as the lengthened $\mathrm{T} 1$ for extended availability of the labeled tracer compared with imaging at low fields (e.g., 1.5 Tesla). The use of 3D GRASE readout after either continuous (33) or pulsed (34) ASL preparation is hence a natural consequence seeking for time-efficient spatial encoding to study brain perfusion (Figure 2). Technical improvements dealing with T2-related point-spread-function blurring, as mentioned before, are documented (35). This could become important because for even higher field strengths such as 7.0 Tesla, the shortening of T2 makes the blurring issue increasingly prominent (36). With shortened readout time provided by 3D GRASE, advanced extensions of ASL to incorporate multiple post-labeling delays to estimate bolus arrival time as in contrast injection studies $(37,38)$ or to measure other related parameters such as water transfer time (39) have become possible. In fact for ASL in the brain, the rapidly increasing utilization of 3D GRASE almost makes it a standard readout module. Applications of ASL with 3D GRASE to other organs also start to appear in 


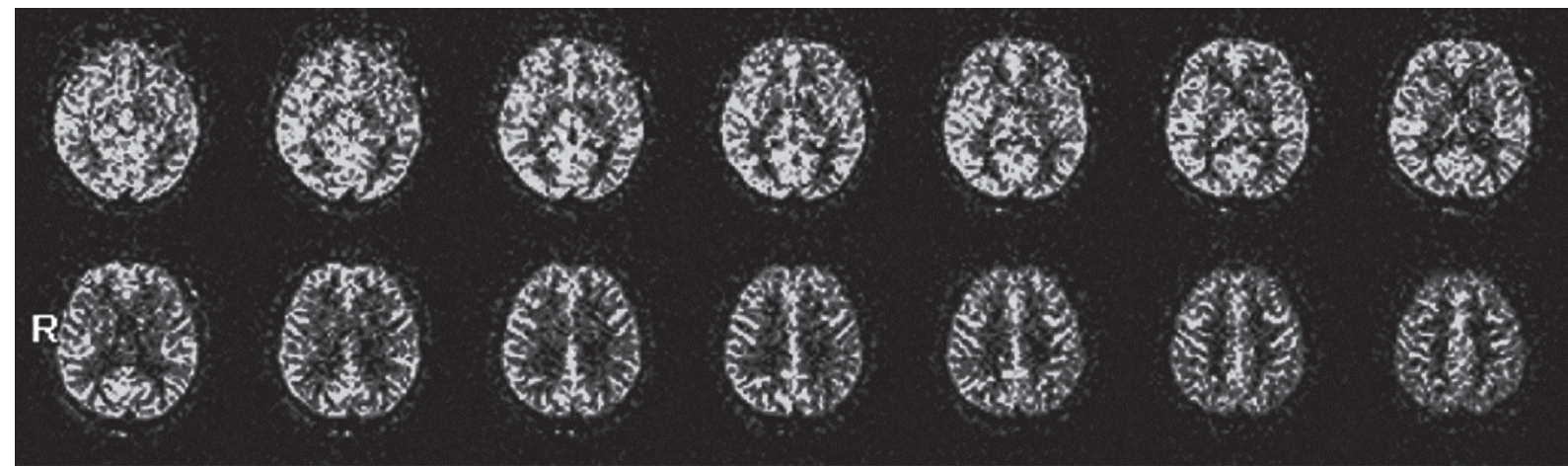

Figure 2 Cerebral blood flow maps obtained using pulsed ASL preparation. The 14 slices shown here were ordered from the inferior to the superior slices (from left to right, from top to bottom). With 3D GRASE readout, the entire volume could be acquired within the scan time of 2.2 minutes, thus facilitating clinical usage when administration of contrast agent is counter-indicated in patients with kidney dysfunction. The greater blood flow in the gray matter than in the white matter was clearly depicted. No point-spread-function-related ghosting or blurring artifacts were visually discernible. ASL, arterial spin labeling; 3D, three-dimensional; GRASE, gradient- and spin-echo.

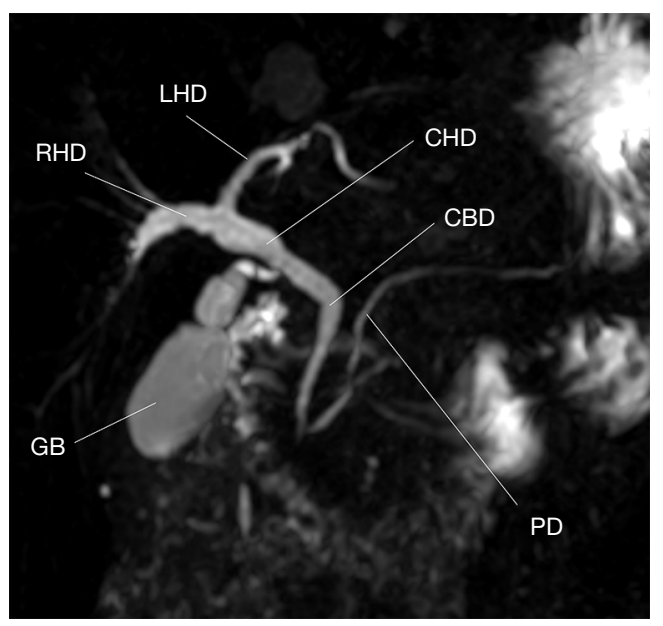

Figure 3 Breath-hold MR cholangiogram acquired at 3.0 Tesla using 3D GRASE from one healthy subject. Within a TR of $262 \mathrm{msec}, 9$ refocusing pulses were applied with 5 gradient-echoes acquired during each echo spacing, resulting in total scan time of 13 seconds after parallel imaging acceleration. In addition to maximum intensity projection provided in this example, $3 \mathrm{D}$ allows depth information to be assessed from the original data set. 3D readout based on FSE would be hampered by SAR limitation of the radiofrequency pulses and the consequent lengthening of scan time prohibiting single breath-hold. MR, magnetic resonance; 3D, three-dimensional; GRASE, gradient- and spin-echo; TR, repetition time; FSE, fast spin-echo; SAR, specific absorption rate; $\mathrm{GB}$, gallbladder; CBD, common bile duct; $\mathrm{CHD}$, common hepatic duct; LHD, left hepatic duct; RHD, right hepatic duct; PD, pancreatic duct. the literature with promising results (40).

Emerging applications of the GRASE sequence are certainly not restricted to perfusion with ASL. Images acquired from the generic GRASE sequence exhibit T2-weighted contrast from the long signal readout (15), which finds several clinical usages such as MR cholangiopancreatography (MRCP). MRCP is conventionally performed using FSE for its excellent immunity to susceptibility artifacts from the airtissue interface in the abdomen. However, as stated before, the SAR issues have placed a limit of the minimum echo spacing for FSE at high fields, leading to long scan time for 3D MRCP based on FSE. Respiratory motion thus becomes the dominant factor determining image quality, which evoked several technical developments such as respiratory gating or navigator correction for free-breathing 3D MRCP with various levels of success (41). For MRCP with FSE in a single breath-hold, one has to accept the alternative of 2D thick-slab MRCP (42), preferably with further acceleration options such as parallel imaging (43). With the highly efficient acquisition from the multiple gradient-echoes, GRASE seems to provide a solution for 3D MRCP at high fields in one single breathhold (Figure 3). Some pilot works on MRCP with 3D GRASE have demonstrated better quality overall than respiratorytriggered 3D FSE, with the superiority mainly attributed to the motion freezing advantage $(26,44)$. Especially for patients with irregular breathing patterns, finishing the scan within one single breath-hold is critically important (45). Susceptibility-related signal loss that may be present due to the gradient-echo nature of the GRASE sequence did not 

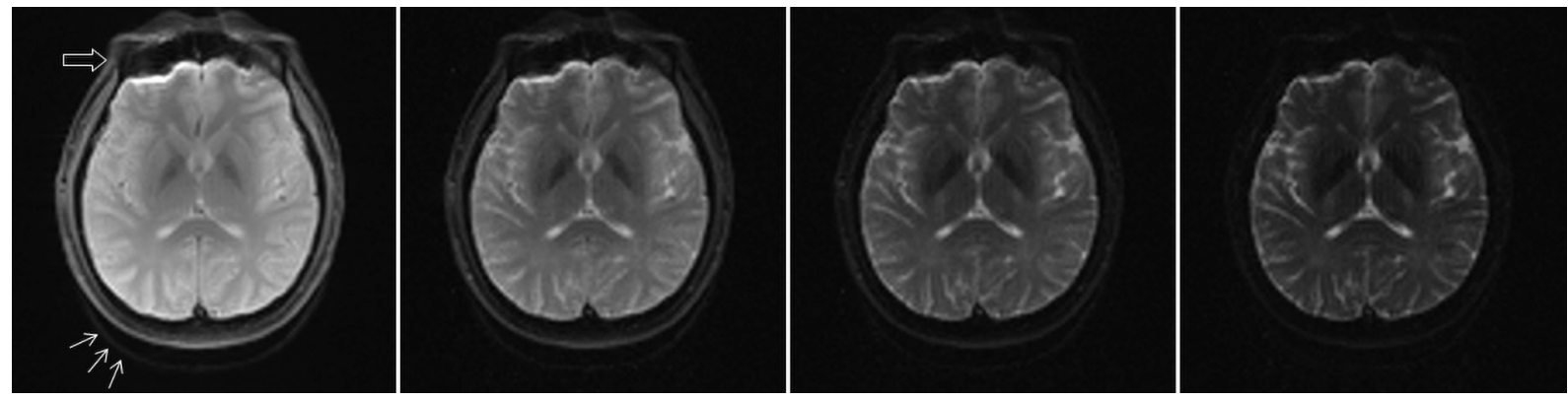

Figure 4 With the aid of parallel imaging, GRASE becomes a multi-EPI sequence where a series of gradient-echoes (four in this example), each acquired within one single echo spacing, are sufficient to form individual EPI images at different TEs (from left to right: 30, 60, 90, and $120 \mathrm{msec}$, respectively). Therefore, T2 mapping is directly achievable in a single shot, as shown in the brain images obtained from a healthy subject. The geometric distortions inherent in EPI acquisitions are evident at the frontal lobe (open arrow), whereas the chemical shift displacement along the phase encoding direction is also seen for the residual subcutaneous fat signals (arrows). EPI, echo-planar imaging.

seem to be of concern (44).

Another application where GRASE is increasingly used nowadays is quantitative relaxation mapping, in particular T2 mapping. Again with its high efficiency in data acquisition, GRASE with different degrees of magnetization preparation allows for estimation of relaxation parameters via the conventional fitting approach. Moreover, with the maturation of parallel imaging using multi-channel receiver arrays, the series of gradient-echoes obtained within one echo spacing interval could actually form one individual EPI image, each at a different spin-echo time (TE) (46). In such a case GRASE turns itself into a multi-EPI sequence whose acquired data are directly available for T2 mapping (Figure 4). Experimental investigations on myocardium have demonstrated effectiveness of this approach (47). Excellent reproducibility of the quantification was reported (48), which is important for the rapidly moving heart, although the T2 values measured using different sequences may not be entirely consistent (49). Myelin water fraction is another example where T2 mapping with GRASE finds usefulness (50). The component of $\mathrm{T} 2$ in the range of $5-20 \mathrm{msec}$, which is believed to arise from the trapped water molecules within the myelin sheath, can be extracted from the signal decaying curve with multiple TE. Technical variations on GRASE to attempt a direct visualization of the myelin water component have also been documented (51).

\section{Conclusions}

Despite of shortcomings reported during the initial development stage, GRASE does exhibit its unique advantages in the modern era of high-field MR systems. Particularly for the $3 \mathrm{D}$ version with appropriate arrangement of the phase encoding order and data processing to improve image quality, the shortened scan time of GRASE allows for imaging within one single breath-hold, hence suitable for abdominal examinations. After further technical advancements, GRASE is anticipated to see clinical applications in even broader areas than those reported in this article. Research investigations such as accurate quantitative relaxation mapping could also be one of the potential fields that may find widened use of GRASE, which warrants exploration by the interested MR scientists.

\section{Acknowledgments}

Funding: The authors received support from the Ministry of Science and Technology under grants MOST 108-2811-E002-552 (Chu ML), MOST 105-2314-B-002-094-MY3 (Wu WC), and MOST 107-2221-E-002-038-MY3 (Chung HW).

\section{Footnote}

Conflicts of Interest: The authors have no conflicts of interest to declare.

\section{References}

1. Le Bihan D. Molecular diffusion nuclear magnetic resonance imaging. Magn Reson Q 1991;7:1-30.

2. Mansfield P, Pykett IL. Biological and medical imaging by NMR. J Magn Reson 2011;213:513-31.

3. Farzaneh F, Riederer SJ, Pelc NJ. Analysis of T2 limitations and off-resonance effects on spatial resolution 
and artifacts in echo-planar imaging. Magn Reson Med 1990;14:123-39.

4. Sekihara K. Steady-state magnetizations in rapid NMR imaging using small flip angles and short repetition intervals. IEEE Trans Med Imaging 1987;6:157-64.

5. Sommer G, Bremerich J, Lund G. Magnetic resonance imaging in valvular heart disease: clinical application and current role for patient management. J Magn Reson Imaging 2012;35:1241-52.

6. Feinberg DA, Oshio K. GRASE (gradient- and spinecho) MR imaging: a new fast clinical imaging technique. Radiology 1991;181:597-602.

7. Oshio K, Feinberg DA. GRASE (Gradient- and spin-echo) imaging: a novel fast MRI technique. Magn Reson Med 1991;20:344-9.

8. Hennig J, Nauerth A, Friedburg H. RARE imaging: a fast imaging method for clinical MR. Magn Reson Med 1986;3:823-33.

9. Oshio K, Feinberg DA. Single-shot GRASE imaging without fast gradients. Magn Reson Med 1992;26:355-60.

10. Feinberg DA, Johnson G, Kiefer B. Increased flexibility in GRASE imaging by k space-banded phase encoding. Magn Reson Med 1995;34:149-55.

11. Johnson G, Feinberg DA, Venkataraman V. A comparison of phase encoding ordering schemes in T2-weighted GRASE imaging. Magn Reson Med 1996;36:427-35.

12. Keller PJ, Karis JP, Fram EK, Heiserman JE, Drayer BP. An alternative to GRASE: toward spin-echo-like contrast with independent reconstruction of gradient-echo images. Magn Reson Med 1996;36:804-8.

13. Fellner F, Schmitt R, Trenkler J, Fellner C, Böhm-Jurkovic $H$. Turbo gradient-spin-echo (GRASE): first clinical experiences with a fast T2-weighted sequence in MRI of the brain. Eur J Radiol 1995;19:171-6.

14. Umek W, Ba-Ssalamah A, Prokesch R, Mallek R, Heimberger K, Hittmair K. Imaging of the brain using the fast-spin-echo and gradient-spin-echo techniques. Eur Radiol 1998;8:409-15.

15. Patel MR, Klufas RA, Shapiro AW. MR imaging of diseases of the brain: comparison of GRASE and conventional spin-echo T2-weighted pulse sequences. AJR Am J Roentgenol 1995;165:963-6.

16. Rockwell DT, Melhem ER, Bhatia RG. GRASE (gradientand spin-echo) MR of the brain. AJNR Am J Neuroradiol 1997;18:1923-8.

17. Melhem ER, Patel RT, Whitehead RE, Bhatia RG, Rockwell DT, Jara H. MR imaging of hemorrhagic brain lesions: a comparison of dual-echo gradient- and spin- echo and fast spin-echo techniques. AJR Am J Roentgenol 1998;171:797-802.

18. Allkemper T, Reimer P, Schuierer G, Peters PE. Study of susceptibility-induced artefacts in GRASE with different echo train length. Eur Radiol 1998;8:834-8.

19. Seidenwurm D, Meng TK, Kowalski H, Weinreb JC, Kricheff II. Intracranial hemorrhagic lesions: evaluation with spin-echo and gradient-refocused MR imaging at 0.5 and 1.5 T. Radiology 1989;172:189-94.

20. Fellner F, Held P, Fellner C, Schmitt R, Obletter N. Fast spin-echo (FSE) and gradient- and spin-echo (GRASE) in fast MRI of the pelvis. Magn Reson Imaging 1997;15:517-24.

21. Postema S, Pattynama PM, van Rijswijk CS, van Erkel A, Tjin A Ton ER. MR imaging of uterine cervical carcinoma: comparison between fast spin-echo MRI and GRASE. Eur Radiol 1998;8:45-9.

22. Dardzinski BJ, Sotak CH, Fisher M, Hasegawa Y, Li L, Minematsu K. Apparent diffusion coefficient mapping of experimental focal cerebral ischemia using diffusionweighted echo-planar imaging. Magn Reson Med 1993;30:318-25.

23. Welch KM, Windham J, Knight RA, Nagesh V, Hugg JW, Jacobs M, Peck D, Booker P, Dereski MO, Levine SR. A model to predict the histopathology of human stroke using diffusion and T2-weighted magnetic resonance imaging. Stroke 1995;26:1983-9.

24. Mitchell DG, Vinitski S, Burk DL Jr, Levy D, Rifkin MD. Variable-flip-angle spin-echo MR imaging of the pelvis: more versatile T2-weighted images. Radiology 1989;171:525-9.

25. Röschmann P. Radiofrequency penetration and absorption in the human body: limitations to high-field wholebody nuclear magnetic resonance imaging. Med Phys 1987;14:922-31.

26. Nam JG, Lee JM, Kang HJ, Lee SM, Kim E, Peeters JM, Yoon JH. GRASE Revisited: breathhold three-dimensional (3D) magnetic resonance cholangiopancreatography using a Gradient and Spin Echo (GRASE) technique at 3T. Eur Radiol 2018;28:3721-8.

27. Nitz WR. Fast and ultrafast non-echo-planar MR imaging techniques. Eur Radiol 2002;12:2866-82.

28. Jovicich J, Norris DG. GRASE imaging at 3 Tesla with template interactive phase-encoding. Magn Reson Med 1998;39:970-9.

29. Trampel R, Reimer E, Huber L, Ivanov D, Heidemann RM, Schäfer A, Turner R. Anatomical brain imaging at 7T using two-dimensional GRASE. Magn Reson Med 
2014;72:1291-301.

30. Mugler JP 3rd. Improved three-dimensional GRASE imaging with the SORT phase-encoding strategy. J Magn Reson Imaging 1999;9:604-12.

31. Kim H, Kim DH, Park J. Variable-flip-angle singleslab 3D GRASE imaging with phase-independent image reconstruction. Magn Reson Med 2015;73:1041-52.

32. Chuang TC, Wu MT, Huang TY, Lin YR, Tsai SY, Chang HC, Chung HW. Stimulated echo induced misestimates on diffusion tensor indices and its remedy. J Magn Reson Imaging 2010;31:1522-9.

33. Fernández-Seara MA, Wang Z, Wang J, Rao HY, Guenther M, Feinberg DA, Detre JA. Continuous arterial spin labeling perfusion measurements using single shot 3D GRASE at 3 T. Magn Reson Med 2005;54:1241-7.

34. MacIntosh BJ, Pattinson KT, Gallichan D, Ahmad I, Miller KL, Feinberg DA, Wise RG, Jezzard P. Measuring the effects of remifentanil on cerebral blood flow and arterial arrival time using 3D GRASE MRI with pulsed arterial spin labelling. J Cereb Blood Flow Metab 2008;28:1514-22.

35. Liang X, Connelly A, Tournier JD, Calamante F. A variable flip angle-based method for reducing blurring in 3D GRASE ASL. Phys Med Biol 2014;59:5559-73.

36. Kemper VG, De Martino F, Yacoub E, Goebel R. Variable flip angle 3D-GRASE for high resolution fMRI at 7 tesla. Magn Reson Med 2016;76:897-904.

37. Qin Q, Huang AJ, Hua J, Desmond JE, Stevens RD, van $\mathrm{Zijl}$ PC. Three-dimensional whole-brain perfusion quantification using pseudo-continuous arterial spin labeling MRI at multiple post-labeling delays: accounting for both arterial transit time and impulse response function. NMR Biomed 2014;27:116-28.

38. Shao X, Liu D, Martin T, Chanlaw T, Devaskar SU, Janzen C, Murphy AM, Margolis D, Sung K, Wang DJJ. Measuring human placental blood flow with multidelay 3D GRASE pseudocontinuous arterial spin labeling at 3T. J Magn Reson Imaging 2018;47:1667-76.

39. Gregori J, Schuff N, Kern R, Günther M. T2-based arterial spin labeling measurements of blood to tissue water transfer in human brain. J Magn Reson Imaging 2013;37:332-42.

40. Nery F, De Vita E, Clark CA, Gordon I, Thomas DL. Robust kidney perfusion mapping in pediatric chronic kidney disease using single-shot 3D-GRASE ASL with optimized retrospective motion correction. Magn Reson Med 2019;81:2972-84.

41. Itatani R, Namimoto T, Kajihara H, Yoshimura A,
Katahira K, Nasu J, Matsushita I, Sakamoto F, Kidoh M, Yamashita Y. Preoperative evaluation of the cystic duct for laparoscopic cholecystectomy: comparison of navigatorgated prospective acquisition correction- and conventional respiratory-triggered techniques at free-breathing $3 \mathrm{D} \mathrm{MR}$ cholangiopancreatography. Eur Radiol 2013;23:1911-8.

42. Schmidt S, Chevallier P, Novellas S, Gelsi E, Vanbiervliet

G, Tran A, Schnyder P, Bruneton JN. Choledocholithiasis: repetitive thick-slab single-shot projection magnetic resonance cholangiopancreaticography versus endoscopic ultrasonography. Eur Radiol 2007;17:241-50.

43. Lee JH, Lee SS, Kim JY, Kim IS, Byun JH, Park SH, Lee MG. Parallel imaging improves the image quality and duct visibility of breathhold two-dimensional thick-slab MR cholangiopancreatography. J Magn Reson Imaging 2014;39:269-75.

44. Yoshida M, Nakaura T, Inoue T, Tanoue S, Takada S, Utsunomiya D, Tsumagari S, Harada K, Yamashita Y. Magnetic resonance cholangiopancreatography with GRASE sequence at 3.0T: does it improve image quality and acquisition time as compared with $3 \mathrm{D}$ TSE? Eur Radiol 2018;28:2436-43.

45. He M, Xu J, Sun Z, Wang S, Zhu L, Wang X, Wang J, Feng F, Xue H, Jin Z. Comparison and evaluation of the efficacy of compressed SENSE (CS) and gradientand spin-echo (GRASE) in breath-hold (BH) magnetic resonance cholangiopancreatography (MRCP). J Magn Reson Imaging 2019. [Epub ahead of print]. doi: 10.1002/ jmri.26863.

46. Chu ML, Chang HC, Oshio K, Chen NK. A singleshot T2 mapping protocol based on echo-split gradientspin-echo acquisition and parametric multiplexed sensitivity encoding based on projection onto convex sets reconstruction. Magn Reson Med 2018;79:383-93.

47. Sprinkart AM, Luetkens JA, Träber F, Doerner J, Gieseke J, Schnackenburg B, Schmitz G, Thomas D, Homsi R, Block W, Schild H, Naehle CP. Gradient Spin Echo (GraSE) imaging for fast myocardial T2 mapping. J Cardiovasc Magn Reson 2015;17:12.

48. Baeßler B, Schaarschmidt F, Stehning C, Schnackenburg B, Giolda A, Maintz D, Bunck AC. Reproducibility of three different cardiac T2-mapping sequences at 1.5T. J Magn Reson Imaging 2016;44:1168-78.

49. Baeßler B, Schaarschmidt F, Stehning C, Schnackenburg B, Maintz D, Bunck AC. A systematic evaluation of three different cardiac T2-mapping sequences at 1.5 and $3 \mathrm{~T}$ in healthy volunteers. Eur J Radiol 2015;84:2161-70.

50. Meyers SM, Kolind SH, MacKay AL. Simultaneous 
measurement of total water content and myelin water fraction in brain at 3T using a T2 relaxation based method. Magn Reson Imaging 2017;37:187-94.

51. Choi JY, Jeong IH, Oh SH, Oh CH, Park NY, Kim HJ, Lee J. Evaluation of normal-appearing white matter

Cite this article as: $\mathrm{Chu} \mathrm{ML}$, Chien $\mathrm{CP}, \mathrm{Wu}$ WC, Chung HW. Gradient- and spin-echo (GRASE) MR imaging: a longexisting technology that may find wide applications in modern era. Quant Imaging Med Surg 2019;9(9):1477-1484. doi: 10.21037/qims.2019.09.13 in multiple sclerosis using direct visualization of short transverse relaxation time component (ViSTa) myelin water imaging and gradient echo and spin echo (GRASE) myelin water imaging. J Magn Reson Imaging 2019;49:1091-8. 